\title{
Statins for the treatment of non alcoholic fatty liver disease in patients with metabolic syndrome
}

Keywords: statin, NAFLD, NASH, metabolic syndrome, treatment

\section{Introduction}

Non-alcoholic fatty liver disease (NAFLD) is the commonest chronic liver disease and is characterized by accumulation of fat $(>5 \%)$ in liver cells in the absence of alcohol abuse, chronic viral hepatitis or other liver disease. ${ }^{1,2}$ NAFLD prevalence in the general population in Western Countries varies from 15 to $30 \%{ }^{3}$ The prevalence of NAFLD rises up to $75-100 \%$ in patients with insulin resistance (IR), such as those with obesity, metabolic syndrome (MetS) or type 2 diabetes mellitus (T2DM). Histological manifestations of NAFLD vary from isolated steatosis to steatohepatitis (NASH), which is characterized by liver necroinflammation and ballooning with or without liver fibrosis, and finally cirrhosis; in some cases NASH may progress to hepatocellular carcinoma. ${ }^{4}$ Data suggests that NASH prevalence ranges from $3-5 \%$ ( $>20 \%$ of NAFLD cases) in the general population, however this rises to approximately $40 \%$ in the morbidly obese and in patients with MetS. ${ }^{4} \mathrm{NAFLD} / \mathrm{NASH}$ is considered as the hepatic manifestation of MetS and is closely related to cardiovascular disease (CVD) risk, ${ }^{5}$ to the extent that NAFLD/NASH and CVD are viewed as different manifestations of the same disease. ${ }^{5,6}$ In the United States (US) National Health and Nutrition Examination Survey (NHANES), which was conducted in 1988-1994 and followed-up more than 11,000 participants for a mean period of 15 years, the degree of liver fibrosis was related with the clinical outcome in NAFLD/NASH patients. ${ }^{6}$ Isolated hepatic steatosis was not related with higher total mortality compared with subjects without NAFLD but there was a considerable and escalating increase in total mortality with increasing levels of liver fibrosis scores as compared with subjects with no fibrosis. ${ }^{6}$ This increase in mortality was mainly due to increased CVD mortality. ${ }^{6}$ As a consequence, NAFLD/NASH with fibrosis have been identified as an independent CVD risk factor. ${ }^{5,6}$

Currently, besides life style measures, which are difficult to be adopted from patients on a long term basis, there is not a generally accepted treatment for NAFLD/NASH and thus there is no medication specifically recommended for the treatment of this disease. ${ }^{7}$ Attention must be paid to the association between NAFLD and CVD, given that these patients are more likely to die due to CVD rather than from liver disease.

In 2010, in a post hoc analysis of the Coronary Heart Disease Evaluation (GREACE) survival study, 437 of the 1,600 patients with overt CVD who were included in the study (the vast majority of them had MetS) had moderately abnormal liver tests at baseline probably due to NAFLD, as this was indicated by liver ultrasound and from the exclusion of alcohol abuse and other liver diseases. ${ }^{8}$ Among these 437 patients, 227 were treated with statins (mainly atorvastatin) and showed a considerable improvement in liver tests $(\mathrm{p}<0.0001)$ whereas 210 participants who did not receive a statin had a further increase of liver enzyme levels. ${ }^{8}$

Statin treatment was safe in patients with CVD and NAFLD;

\author{
Volume 3 Issue I - 2016
}

\author{
Vasilios G Athyros, Konstantinos Tziomalos, \\ Asterios Karagiannis \\ Department of Internal Medicine, Aristotle University of \\ Thessaloniki, Greece
}

Correspondence: Vasilios G Athyros, Second Propedeutic Department of Internal Medicine, Medical School, Aristotle University of Thessaloniki, Hippocration Hospital, 55I32, Thessaloniki, Greece, Tel +30 2310892 606, Fax +302310 835 955,Email vathyros@gmail.com

Received: December 31, 2015 | Published: January 09, 2016

only $1 \%$ discontinued treatment. ${ }^{8}$ Thus, atorvastatin did not have any adverse effect on liver enzymes; on the contrary, it reduced them substantially and improved liver ultrasound within the 3year followup period of the study. ${ }^{8}$ Moreover, atorvastatin-treated patients had a substantial reduction in recurrent CVD events (68\% vs usual care) during the 3year follow-up period ( $\mathrm{p}=0.007$ compared with the $39 \%$ reduction in CVD events in patients with normal liver enzymes). ${ }^{8}$ This finding suggests that patients who are commonly deprived of statin treatment because of liver enzyme elevations had greater clinical benefit from CVD event reduction than those with normal liver structure and function. ${ }^{8}$

More recently, a post hoc analysis of the Assessing the Treatment Effect in Metabolic Syndrome without Perceptible Diabetes (ATTEMPT) study $(\mathrm{n}=1,123)$, that included only patients with MetS but without overt CVD or T2DM (i.e. primary prevention), investigated the effect of atorvastatin-based multifactorial intervention on NAFLD diagnosed with Ultrasonography. ${ }^{9}$ The results showed that there was a complete normalization of liver enzyme values within 42 months and that there were no CVD events in patients with LDL-C levels $<100 \mathrm{mg} / \mathrm{dl}$ (mean atorvastatin dose $34 \mathrm{mg} / \mathrm{dl}$ ) for the duration of the study, while there were 5 non-fatal CVD events in the lower atorvastatin dose (mean $24 \mathrm{mg} /$ day) group $(\mathrm{p}=0.024) .{ }^{9}$

These results of GREACE and ATTEMPT studies were confirmed in 2013 by a post hoc analysis of the Incremental Decrease through Aggressive Lipid Lowering [IDEAL] study $(n=8,863) .{ }^{10}$ IDEAL showed a nearly double reduction in 5year CVD event rate with atorvastatin $80 \mathrm{mg} /$ day in patients with NAFLD compared with 20 $40 \mathrm{mg}$ of simvastatin. ${ }^{10}$ Moreover, the high atorvastatin dose $(80 \mathrm{mg} /$ day) induced the greatest reduction in liver enzyme concentrations during the study. ${ }^{10}$

The criticism on the results above was focused to the fact that NAFLD/NASH was not verified by liver biopsies, before and after treatment. For this reason we designed a pilot study including liver biopsies in patients with MetS, elevated liver enzymes and definite NASH in biopsy (fibrosis, necroinflammation, ballooning, and steatosis. ${ }^{11}$ In 2014, preliminary results in 6 patients showed that $10 \mathrm{mg} /$ day of rosuvastatin monotherapy induced within 1year a complete 
normalization of lipid profile, a resolution of MetS, a normalization of all liver enzymes, and complete resolution of NASH in the repeat biopsy, which revealed normal liver histology. ${ }^{11}$
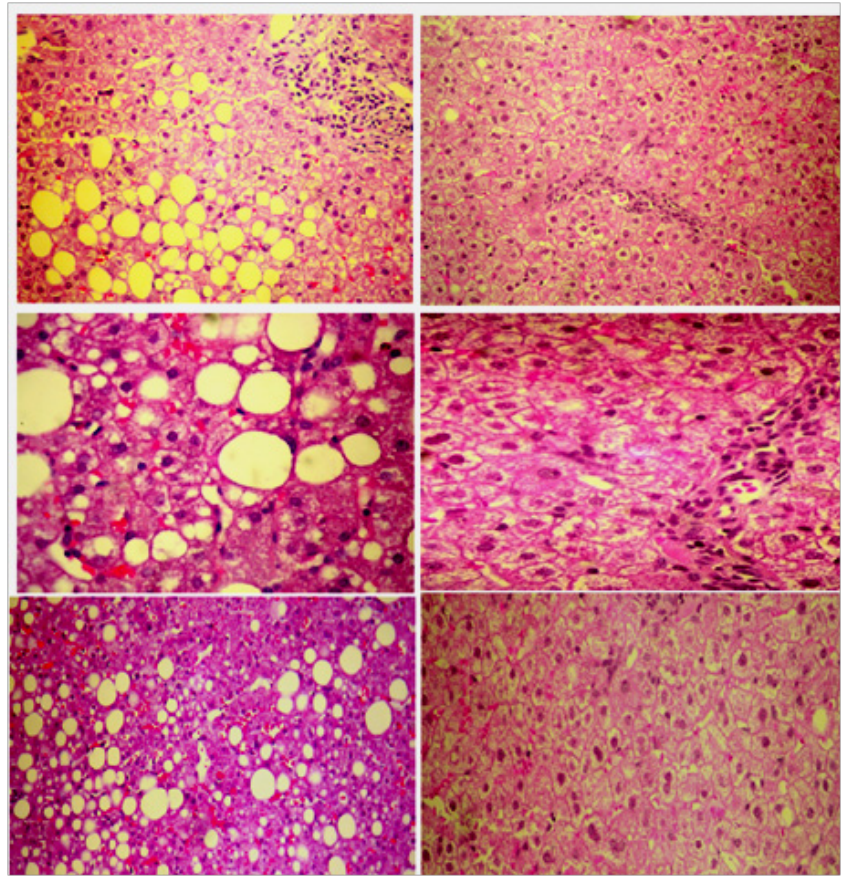

Figure I Liver biopsies from people with MetS, at baseline in the left panel (showing steatosis, hepatocyte ballooning degeneration, diffuse lobular mixed acute and chronic inflammation, and perivenular, perisinusoidal collagen disposition) and after one year of rosuvastatin monotherapy with $10 \mathrm{mg} /$ day in the right panel (showing normal liver histology).

In 2015, we reported the final results in 20 patients with MetS and biopsy-proven NASH, which were identical with those of the pilot study. ${ }^{12}$ At the end of the 1year follow-up, patients did not have MetS anymore, because of a reduction in triglyceride levels, an increase in high density lipoprotein cholesterol levels, and an unexpected reduction in fasting plasma glucose by the "diabetogenic" rosuvastatin. ${ }^{12}$ It seems that the resolution of NASH by rosuvastatin ameliorated insulin resistance in these patients with MetS. Importantly, waist circumference and the body mass index did not change and therefore the improvement in glucose metabolism cannot be attributed to the reduction of abdominal obesity. ${ }^{12}$

\section{Conclusion}

There is no generally accepted treatment for NAFLD/NASH. The early detection of NAFLD/NASH and particularly of patients with advanced liver fibrosis, which exacerbates CVD risk, is crucial in patients with MetS. Along with life style changes, atorvastatin or rosuvastatin treatment (given that there are no data for other statins) is safe even at high doses and might be an effective solution for the treatment of NAFLD/NASH (at least in those with dyslipidaemia or MetS), might lead to its resolution and it will substantially reduce CVD risk, related to NASH, both in primary or secondary prevention..$^{8-10,13}$ We should not deprive NAFLD/NASH patients from a life saving treatment. This problem may include up to a billion subjects worldwide and it is too big to ignore.

\section{Acknowledgements}

None.

\section{Conflict of interest}

This review was written independently. The authors did not receive financial or professional help with the preparation of the manuscript. The authors have no conflict of interest.

\section{References}

1. Baran B, Akyüz F. Non-alcoholic fatty liver disease: what has changed in the treatment since the beginning? World $J$ Gastroenterol. 2014;20(39):14219-14229.

2. Vernon G, Baranova A, Younossi ZM. Systematic review: the epidemiology and natural history of non-alcoholic fatty liver disease and non-alcoholic steatohepatitis in adults. Aliment Pharmacol Ther. 2011;34(3):274-285.

3. Angulo P. GI epidemiology: nonalcoholic fatty liver disease. Aliment Pharmacol Ther. 2007;25(8):883-889.

4. Jiang $\mathrm{CM}, \mathrm{Pu} \mathrm{CW}$, Hou $\mathrm{YH}$, et al. Non alcoholic steatohepatitis a precursor for hepatocellular carcinoma development. World $J$ Gastroenterol. 2014;20(44):16464-16473.

5. Fargion S, Porzio M, Fracanzani AL. Nonalcoholic fatty liver disease and vascular disease: state-of-the-art. World J Gastroenterol. 2014;20(37):13306-13324.

6. Kim D, Kim WR, Kim HJ, et al. Association between noninvasive fibrosis markers and mortality among adults with nonalcoholic fatty liver disease in the United States. Hepatology. 2013;57(4):1357-1365.

7. Munteanu MA, Mircea PA. From NAFLD to Cardiovascular Disease. Is it (Still) the Metabolic Syndrome? Clujul Med. 2014;87(2):80-86.

8. Athyros VG, Tziomalos K, Gossios TD, et al. Safety and efficacy of long-term statin treatment for cardiovascular events in patients with coronary heart disease and abnormal liver tests in the Greek Atorvastatin and Coronary Heart Disease Evaluation (GREACE) Study: a post-hoc analysis. Lancet. 2010;376(9756):1916-1922.

9. Athyros VG, Giouleme O, Ganotakis ES, et al. Safety and impact on cardiovascular events of long-term multifactorial treatment in patients with metabolic syndrome and abnormal liver function tests: a post hoc analysis of the randomized ATTEMPT study. Arch Med Sci. 2011;7(5):796-805.

10. Tikkanen MJ, Fayyad R, Faergeman O, et al. Effect of intensive lipid lowering with atorvastatin on cardiovascular outcomes in coronary heart disease patients with mild-to-moderate baseline elevations in alanine aminotransferase levels. Int J Cardiol. 2013;168(4):3846-3852.

11. Kargiotis K, Katsiki N, Athyros VG, et al. Effect of rosuvastatin on non-alcoholic steatohepatitis in patients with metabolic syndrome and hypercholesterolaemia: a preliminary report. Curr Vasc Pharmacol. 2014;12(3):505-511.

12. Kargiotis K, Athyros VG, Giouleme O, et al. Resolution of non-alcoholic steatohepatitis by rosuvastatin monotherapy in patients with metabolic syndrome. World J Gastroenterol. 2015;21(25):7860-7868.

13. Mihaylova B, Emberson J, Blackwell L, et al. The effects of lowering LDL cholesterol with statin therapy in people at low risk of vascular disease: meta-analysis of individual data from 27 randomized trials. Lancet. 2012;380(9841):581-590. 